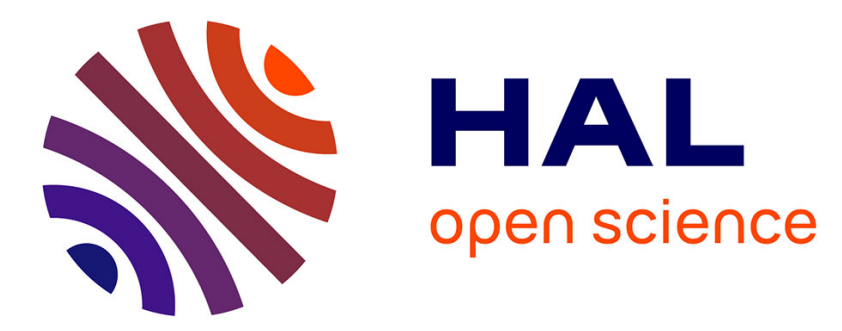

\title{
Sleepers Selectively Suppress Informative Inputs during Rapid Eye Movements
}

Matthieu Koroma, Célia Lacaux, Thomas Andrillon, Guillaume Legendre, Damien Léger, Sid Kouider

\section{- To cite this version:}

Matthieu Koroma, Célia Lacaux, Thomas Andrillon, Guillaume Legendre, Damien Léger, et al.. Sleepers Selectively Suppress Informative Inputs during Rapid Eye Movements. Current Biology - CB, 2020, 30 (12), pp.2411-2417. 10.1016/j.cub.2020.04.047 . hal-03015896

\section{HAL Id: hal-03015896 https://hal.science/hal-03015896}

Submitted on 20 Nov 2020

HAL is a multi-disciplinary open access archive for the deposit and dissemination of scientific research documents, whether they are published or not. The documents may come from teaching and research institutions in France or abroad, or from public or private research centers.
L'archive ouverte pluridisciplinaire HAL, est destinée au dépôt et à la diffusion de documents scientifiques de niveau recherche, publiés ou non, émanant des établissements d'enseignement et de recherche français ou étrangers, des laboratoires publics ou privés. 


\section{Current Biology}

\section{Sleepers Selectively Suppress Informative Inputs during Rapid Eye Movements}

\section{Highlights}

- A neural decoder tracks speech processing in a cocktailparty paradigm during sleep

- Speech is encoded in cortical activity during rapid eye movement (REM) sleep

- Informative speech is selectively processed over meaningless speech during REM sleep

- Informative speech is on the contrary selectively suppressed during eye movements within REM

\section{Authors}

Matthieu Koroma, Célia Lacaux, Thomas Andrillon, Guillaume Legendre, Damien Léger, Sid Kouider

\section{Correspondence}

sid.kouider@ens.fr

\section{In Brief}

Sleepers are sensitive to stimuli during rapid eye movement (REM) sleep, yet how they protect internal activity from external interference is poorly described. Koroma et al. show that informative signals are overall selectively amplified during REM sleep but conversely selectively suppressed during eye movements, a marker of dreaming activity. 


\title{
(nort \\ Sleepers Selectively Suppress Informative Inputs during Rapid Eye Movements
}

\author{
Matthieu Koroma, ${ }^{1,2}$ Célia Lacaux, ${ }^{2,3,4,8}$ Thomas Andrillon, ${ }^{5,8}$ Guillaume Legendre, ${ }^{6}$ Damien Léger, ${ }^{7}$ and Sid Kouider ${ }^{1,9, *}$ \\ ${ }^{1}$ Brain and Consciousness Group (ENS, EHESS, CNRS), Département d'Études Cognitives, École Normale Supérieure - PSL Research \\ University, 75005 Paris, France \\ 2Sorbonne Université, École Doctorale Cerveau Cognition Comportement, Université Pierre et Marie Curie, 9 Quai Saint Bernard, 75005 Paris, \\ France \\ 3Sorbonne University, IHU@ICM, INSERM, CNRS UMR7225, 75013 Paris, France \\ ${ }^{4}$ AP-HP, Hôpital Pitié-Salpêtrière, Service des Pathologies du Sommeil, 75013 Paris, France \\ ${ }^{5}$ School of Psychological Sciences and Turner Institute for Brain and Mental Health, Monash University, Melbourne, VIC 3800 , Australia \\ ${ }^{6}$ Department of Neuroscience, Faculty of Medicine, University of Geneva, 1211 Geneva, Switzerland \\ 7 Université de Paris, Paris Descartes, APHP, Hôtel Dieu, Centre du Sommeil et de la Vigilance et EA 7330 VIFASOM, 75004 Paris, France \\ ${ }^{8}$ These authors contributed equally \\ 'Lead Contact \\ *Correspondence: sid.kouider@ens.fr \\ https://doi.org/10.1016/j.cub.2020.04.047
}

\section{SUMMARY}

Sleep leads to a disconnection from the external world. Even when sleepers regain consciousness during rapid eye movement (REM) sleep, little, if any, external information is incorporated into dream content [13]. While gating mechanisms might be at play to avoid interference on dreaming activity [4], a total disconnection from an ever-changing environment may prevent the sleeper from promptly responding to informative events (e.g., threat signals). In fact, a whole range of neural responses to external events turns out to be preserved during REM sleep [5-9]. Thus, it remains unclear whether external inputs are either processed or, conversely, gated during REM sleep. One way to resolve this issue is to consider the specific impact of eye movements (EMs) characterizing REM sleep. EMs are a reliable predictor of reporting a dream upon awakening [10,11], and their absence is associated with a lower arousal threshold to external stimuli [12]. We thus hypothesized that the presence of EMs would selectively prevent the processing of informative stimuli, whereas periods of REM sleep devoid of EMs would be associated with the monitoring of external signals. By reconstructing speech in a multi-talker environment from electrophysiological responses, we show that informative speech is amplified over meaningless speech during REM sleep. Yet, at the precise timing of EMs, informative speech is, on the contrary, selectively suppressed. These results demonstrate the flexible amplification and suppression of sensory information during REM sleep and reveal the impact of EMs on the selective gating of informative stimuli during sleep.

\section{RESULTS}

We used a neural decoding approach allowing us to track the selective processing of speech in a cocktail-party paradigm. Participants $(N=18)$ heard two competing auditory streams while their electroencephalogram (EEG) was recorded. One stream, played in one ear, was meaningful (e.g., informative speech), while the other stream, played in the other ear, had normal syntactic and phonological properties but was meaningless (i.e., Jabberwocky speech as in Lewis Carroll's Jabberwocky poem). Participants were asked to focus only on informative speech and ignore Jabberwocky speech (Figure 1). They first performed this task in wakefulness and were then allowed to sleep during a morning nap, at times of high rapid eye movement (REM) sleep pressure (STAR Methods). We tracked speech processing by using a stimulus reconstruction approach, which allows the continuous reconstruction of a sound's envelope based on its neural responses [13, 14]. We showed previously that selective orientation toward either informative or Jabberwocky speech could be decoded from scalp EEG using this technique [15]. When participants are instructed to focus only on the informative stream and then enter non-REM (NREM) sleep, EEG markers reveal that informative speech is overall still enhanced, but it is specifically suppressed during sleep slow waves, a hallmark of NREM sleep [15]. Here, we used the same approach to evaluate whether informative speech was still preferentially processed during REM sleep and, in particular, whether the presence of eye movements (EMs) plays a role in gating the processing of external information.

We first found that both informative speech and Jabberwocky speech could be reconstructed across wakefulness, light NREM sleep, and REM sleep, revealing preserved auditory encoding across vigilance states (Figure $2 A ; p<0.05$ for all conditions corrected for multiple comparisons). We replicated, with this independent dataset, our previous results that informative speech was preferentially reconstructed over Jabberwocky speech in wakefulness and light NREM sleep (informative versus 
TRAINING

Instruction: Listen to the stories in both ears

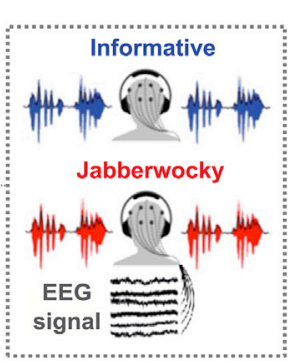

Sound envelope

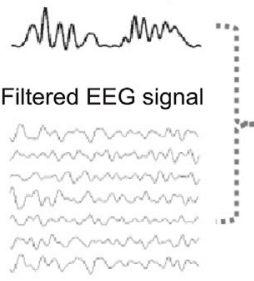

WAKE TEST

Focus on Informative and ignore Jabberwocky

\section{SLEEP TEST}

Novel stories played during sleep

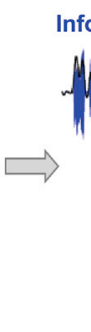

Informative
Informative Jabberwocky Jabberwocky
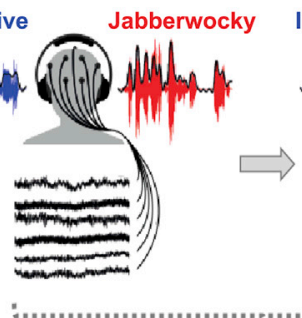

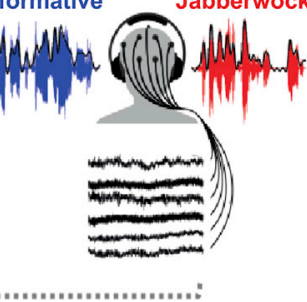

Linear model

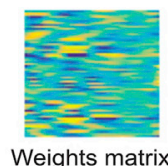

Reconstructed envelope whis

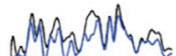
$R=0.04$ Correlation with each envelope $\mathrm{R}=0.01$

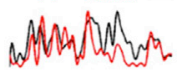

$\mathrm{R}_{\text {Informative }}>\mathrm{R}$ Jabberwocky $=$ Correct trial

Figure 1. Morning Nap Cocktail-Party Paradigm

First, participants listened to 12 stories in both ears, 6 informative stories followed by 6 Jabberwocky stories (training phase). Jabberwocky stories have normal syntactic and phonological structures but are made of novel/meaningless words. Then, participants were instructed to focus on the informative story that was played in one ear while ignoring the Jabberwocky story that was played in the other ear (test phase). After 8 trials during which participants had to perform the task in wakefulness (wake test phase), participants were allowed to fall asleep while the trials from the wake test phase were continuously played (sleep test phase: 90 min; see Figure S4 for two examples of hypnograms and Table S1 for sleep statistics). Novel trials with new informative (blue) and Jabberwocky (red) stories were played whenever participants were sleeping. To track speech processing using cerebral activity during the test phase, training trials were first used to map the EEG response to variations of the sound envelope. The obtained linear model was then used to reconstruct a sound envelope from the filtered EEG recorded during wake and sleep test trials. A correlation score was computed by comparing the

predicted sound envelope with the envelope of each auditory stream. Trials with a higher reconstruction score for the informative speech compared to the Jabberwocky speech were scored as correct and the percentage of correct trials per condition defined the decoding performance.
Jabberwocky for wake, effect size, $r=0.86, p<0.001$; light NREM sleep, $r=0.51, p<0.05$; Figure $2 \mathrm{~A}$ ). We show additionally that these results extend to REM sleep (informative versus Jabberwocky, $r=0.65, p<0.01$; Figure $2 \mathrm{~A}$ ). These results are unlikely to be attributed to a bias in our decoding model as both informative and Jabberwocky speech were equally reconstructed when presented alone in both ears during wakefulness (informative versus Jabberwocky, non-significant, Bayes factor: 3.80; Figure S1B). We then computed a decoding score corresponding to the proportion of trials with a higher reconstruction score for informative speech compared to Jabberwocky speech. We found that decoding performance was significantly above the chance level of $50 \%$ for both wakefulness and REM sleep (wake, $r=$ $0.83, p<0.001$; REM, $r=0.61, p<0.01$; Figure 2B). Yet, decoding performance was lower in REM sleep than during wakefulness (wake versus REM sleep, $r=0.81, p<0.001$; Figure 2B).

To investigate whether this difference resulted from the disruption of auditory encoding for all streams or more specifically from informative speech, we compared reconstruction scores for informative and Jabberwocky speech separately. We found that informative speech and Jabberwocky speech are differently affected across wakefulness and REM sleep $(\mathrm{t}(677.35)=-4.41, \mathrm{p}<0.001$ for the interaction informative versus Jabberwocky $\times$ wake versus REM sleep). Post hoc analyses revealed that the reconstruction of informative speech was selectively modulated by the transition to REM sleep (wake versus REM sleep, $r=0.68, p<0.01$; Figure $2 \mathrm{C}$ ), while Jabberwocky speech remained unaffected (wake versus REM sleep, non-significant, Bayes factor: 3.64; Figure 2C). Thus, decreased decoding performance during REM sleep appears linked to a selective reduction in processing informative speech, rather than a general decline in the encoding of auditory signals.
We thus tested whether EMs, a marker of oneiric activity that occurs during REM sleep, are associated with the selective gating of informative speech. We first removed EM-related activity from EEG using independant component analysis (ICA) to avoid muscular contamination of speech reconstruction (STAR Methods). Our analyses revealed an inverse correlation between the amount of EMs per trial and the reconstruction of informative speech $(p<0.001$; Figure $3 A)$. Crucially, this was not the case for Jabberwocky speech ( $p>0.05$; Figure $3 B$ ). This contrast between informative and Jabberwocky speech is not due to a floor effect in the reconstruction of Jabberwocky speech, which was still above chance, albeit at a lower level than informative speech. It suggests that EMs do not affect the processing of Jabberwocky speech and additionally reinforces the view that artifacts caused by EMs do not reduce the quality of stimulus reconstruction. It also suggests that the presence of EMs selectively modulates the processing of informative signals. To confirm this interpretation, we investigated the temporal relationship between the occurrence of EMs and the selective gating of informative speech, by performing a time-resolved analysis of reconstruction scores around EMs. Within a $10 \mathrm{~s}$ window around the onset of EMs, we found that informative speech was amplified before EMs $\left([-7.8,-2.8] \mathrm{s}, \mathrm{d}=0.63, \mathrm{P}_{\text {cluster }}<\right.$ 0.05 ), but this effect disappeared during and after EMs (Figure $3 \mathrm{C}$ ). Contrastingly, no such modulation could be evidenced for Jabberwocky speech. As EMs can occur in bursts, the reconstruction around a given EM may be impacted by the presence of surrounding EMs. To test for this potential contamination, we investigated the time course of the reconstruction score at the beginning and end of burst EMs. Our analysis revealed that the amplification of informative speech was present before the beginning of bursts and then disappeared at their onsets $([-4.6,-0.2] \mathrm{s}, \mathrm{d}=1.48$, $\mathrm{p}<0.001$; Figure S2A). Conversely, we observed that the 
A
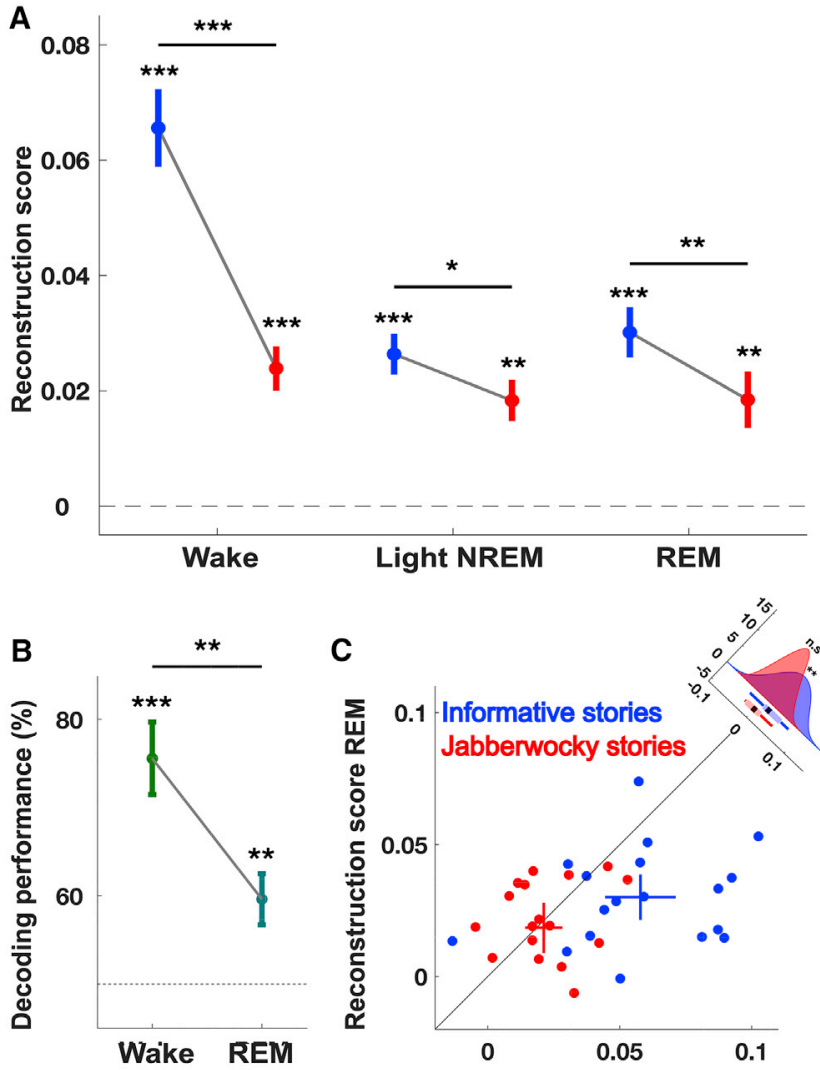

confirmed that the presence of isolated EMs was indeed modulating the selective processing of informative speech $(t(1180)=-3.52, p<0.001$ for the interaction informative versus Jabberwocky $\times$ isolated EM versus REM sleep; Figure 4A). Post hoc analyses revealed that informative speech was overall selectively amplified during REM sleep but selectively suppressed at the onset of isolated EMs (informative versus Jabberwocky in REM sleep, $r=0.52, p<0.05$; during isolated Ems, $r=-0.67$, $p<0.05$; informative versus 0 during REM sleep, $r=0.86, p<$ 0.01; Jabberwocky versus 0 during REM sleep, $r=0.63, p<$ 0.05 ; Figure $4 \mathrm{~A}$ ). We then confirmed that such selective suppression of the informative stream was restricted to the period surrounding the onset of isolated EMs $([-2.6,1.5] \mathrm{s}, \mathrm{d}=-0.73$, $\mathrm{P}_{\text {cluster }}<0.05$; Figure S2C). We further checked that removing eye-movement artifacts with ICA was not responsible for our effects and we obtained similar results with and without the ICA procedure (Figure S3). These analyses confirm that the presence of EMs is tightly associated with the selective suppression of informative speech.

We finally inspected more systematically the distinction between tonic (tREM) and phasic (pREM) REM sleep. Temporal windows of $20 \mathrm{~s}$ were scored as pREM sleep in the presence of bursts of EMs, and as tREM sleep otherwise. In agreement with the literature $[16,17]$, we obtained $\sim 20 \%$ of pREM sleep (Table S1). Congruent with the results above, we found that the selective processing of informative speech is distinct during $p R E M$ and tREM sleep $(t(1198)=-2.07, p<0.05$ for the interaction tonic versus phasic REM $\times$ informative versus Jabberwocky speech; Figure 4B). Post hoc analyses revealed that the selective amplification of informative speech was present during tREM sleep (informative versus Jabberwocky, $r=0.55, p<0.05$ ) but absent during pREM sleep (not-significant, Bayes factor: 5.36). These analyses confirm that periods of sustained eye-movement activity prevent the preferential processing of informative speech. Wakefulness and Sleep

(A) The reconstruction scores for informative (blue) and Jabberwocky (red) speech reveal preserved encoding of auditory speech and a selective amplification of the informative speech across wake, light NREM sleep, and REM sleep. Mean and SEM across participants are represented as filled circles and solid vertical lines, respectively. Stars show significance levels against 0 and differences across story types for each vigilance state $\left({ }^{\star \star *} p<0.001,{ }^{* *} p<0.01\right.$, $\left.{ }^{*} \mathrm{p}<0.05\right)$. See also Figures S1C-S1E.

(B) Decoding performances show reduced selective amplification of informative stories during REM sleep compared to wake. Mean and SEM across participants are represented as respectively filled circles and solid vertical lines. Stars show significance levels against chance (dotted line, 50\% for each vigilance state) and across vigilance state $\left({ }^{* * *} p<0.001,{ }^{* *} p<0.01,{ }^{*} p<0.05\right)$. (C) Reconstruction scores show a selective modulation of informative speech between wake and REM sleep that is absent for the Jabberwocky speech. Each data point represents the reconstruction scores for each story type across vigilance states for each individual subject $(N=18)$. Solid lines depict SEM and are centered on the average across subjects for each story type. Distribution of the difference between vigilance states for each story type are modeled by a Gaussian curve and are represented in the top right corner. Solid black lines represent the mean, shaded areas mark the first and third quartiles, and solid lines represent the whiskers. Stars show significance levels against $0\left({ }^{\star \star \star} p<0.001,{ }^{\star \star} p<0.01,{ }^{\star} p<0.05\right)$.

amplification of informative speech was regained only after burst offsets $([5.0,7.5] \mathrm{s}, \mathrm{d}=0.98, \mathrm{p}<0.05$; Figure S2B).

We then refined our analysis by inspecting isolated EMs (i.e., with no more than one additional EM nearby). We compared reconstruction scores obtained at the onset of isolated EMs with that computed on the entire REM sleep period. We

\section{DISCUSSION}

Our study first confirms that auditory stimuli are still processed by the sleeping brain, in line with results showing a preservation of neural responses to external sounds in the auditory cortex during REM sleep [5, 6]. They further add evidence that salient stimuli are selectively processed during REM sleep [7-9, 18, 19]. More crucially, presenting competing auditory streams allowed us to show that informative stimuli can be flexibly enhanced or suppressed depending on the presence of EMs. Such results are consistent with previous reports showing that responses to auditory stimulation and deviant tones are reduced in the presence of EMs during REM sleep [20,21], as well as the elevation of arousal thresholds during phasic compared to tonic REM sleep [12]. By showing nevertheless that this stimulus gating did not affect the encoding of meaningless speech during REM sleep, we provide first evidence of a selectivity of sensory suppression mechanisms during EMs.

While the mechanisms underlying sensory disconnection have been relatively well studied during NREM sleep, such investigations have been so far limited for REM sleep [4]. It was first proposed that a gating mechanism would prevent auditory information from being transmitted to higher-order associative regions during sleep, but, to date, such cortical 
A

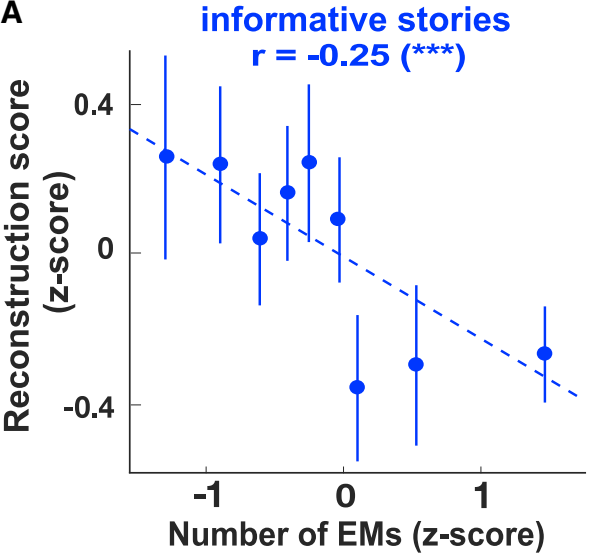

B

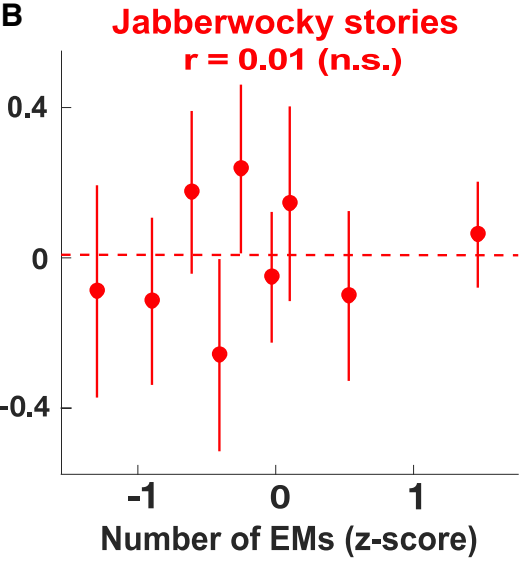

C

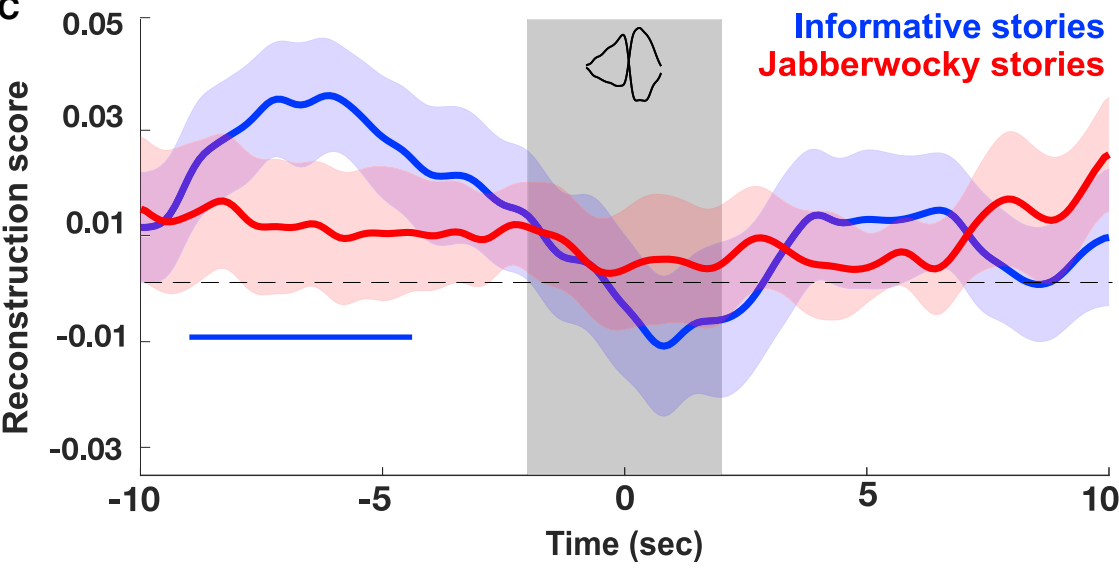

Figure 3. Selective Suppression of Informative Speech in the Presence of Eye Movements

(A and B) The amount of eye movements (EMs) per trial was negatively correlated with the reconstruction scores for the informative (A) but not for the Jabberwocky (B) speech. Values were $Z$ scored for each participant $(N=18)$ and binned for visual purposes ( $\mathrm{n}=10$ bins on the sorted number of EMs). Mean and SEM across participants are represented as respectively filled circles and solid vertical lines. The dotted line indicates the linear regression fit between variables, and significance of Pearson's correlation scores is reported ${ }^{* \star *} p<$ $0.001)$.

(C) Time course of the reconstruction score around the onset of EMs shows a drop in the selective processing of the informative speech during EMs. Reconstruction scores of each auditory stream were computed on $4 \mathrm{~s}$ sliding windows with 100 -ms steps from $-10 \mathrm{~s}$ to $+10 \mathrm{~s}$ around the onset of EMs $(t=0 \mathrm{~s})$. Mean and SEM are represented respectively with solid lines and shaded areas. Blue horizontal line denotes significant clusters of reconstruction scores differing from 0 for informative speech $(p<0.05$ after cluster correction; STAR Methods). See also Figures S2 and S3A.

suppression of informative speech while leaving the Jabberwocky speech unaffected. Such selective suppression may reflect the presence of ongoing high-order internal activity, such as dreaming.

gating has not been evidenced in REM sleep [6]. Other studies alternatively suggested that endogenous activations, potentially associated with the generation of dreams, could interfere with the processing of external events [4]. In the present study, we tracked the neural processing of two competing streams differing in terms of meaningfulness. The selective processing of meaningful stories during REM sleep suggests that associative regions are also involved in the selection of stimuli based on their informative content, ruling out the notion of a thalamic [22] or cortical [23] gating of external inputs during REM sleep. However, attention may also be impaired during REM sleep, as observed in other altered states of consciousness such as hypnosis [4]. Here, we found that selective amplification of informative speech was overall maintained during REM sleep, even if reduced compared to wakefulness. This suggests that attentional resources might, at least partially, be preserved across vigilance states. Because EMs are closely associated with internal activity, we propose that our results buttress the "informational gating" hypothesis, i.e., a competition between the processing of internal information versus external information during REM sleep [4, 24].

The "informational gating" hypothesis suggests a pivotal role for selective processing to balance internal versus external demands depending on stimulus properties (e.g., informative stimuli) and spontaneous activity (e.g., dreaming activity). Representations of competing speeches are encoded separately in the auditory cortex [25], allowing in our study for the selective
Indeed, substantial literature investigating neural processes during eye movements indicates a link between EMs and dreams (see [26] for a recent review) [10, 26-32]. Yet, dreaming has also been reported outside EMs, during both REM and NREM sleep [33, 34]. Assessing the impact of other markers of dreaming activity (e.g., EEG spectral changes [35]) on sensory processing should allow investigating whether our results generalize to other correlates of dreaming or whether they are specific to the occurrence of EMs.

Functional neuroimaging studies indicate a competition between internal and external processing during EMs. Indeed, higher-order cortices associated with spontaneous activity correlate negatively with the activity of sensory areas during REM sleep, and a thalamo-cortical network may favor internal activity at the expense of sensory processing [21, 36, 37]. By blocking the processing of salient information-known to be more susceptible to wake up the sleeper [7, 38]-suppressive mechanisms of sensory stimuli may endorse the role of sleep protection, preventing informative signals from interfering with internal activity. Here, we hypothesized that meaningless signals would be considered as background noise and thus would not interfere with internal processes, such as memory consolidation or dreaming activity. Our results support this hypothesis, but it remains unsettled whether Jabberwocky stories would also be suppressed during EMs if they were presented alone rather than in competition with informative stories. However, even if such a suppression were observed, it would not 
A

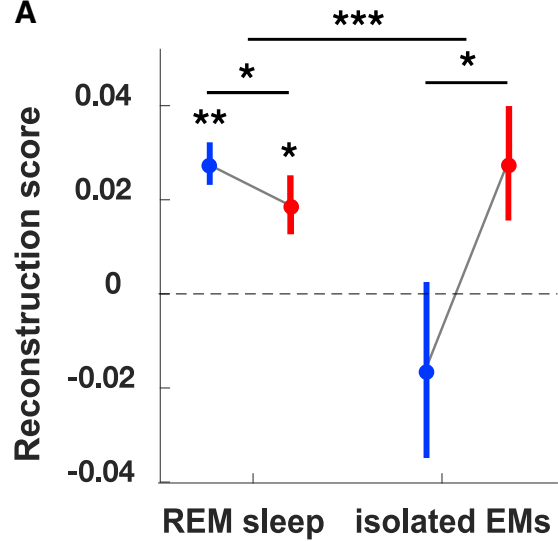

B

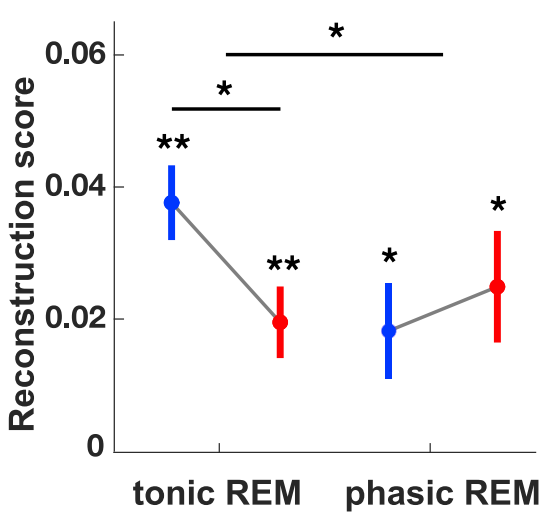

Figure 4. Flexible Selective Processing Depends on the Presence of Eye Movements (A) Informative speech is selectively amplified during REM sleep but selectively suppressed at the onset of isolated EMs. Reconstruction scores are computed on $4 \mathrm{~s}$ windows either on the entire REM period or centered on the onset of isolated EMs. Mean and SEM across participants $(\mathrm{N}=14)$ are represented as respectively filled circles and solid vertical lines. Stars show significant differences against 0 and interactions across story types and conditions $\left({ }^{* * *} p<0.001\right.$, $\left.{ }^{* *} p<0.01,{ }^{*} p<0.05\right)$. See also Figures S2C and S3B.

(B) Informative speech is selectively amplified in windows without bursts of EMs (tonic REM sleep, $\mathrm{N}=18$ ) but not in the presence of bursts of EMs (phasic REM sleep, $N=8$ ). Mean and SEM of reconstruction scores across participants are represented respectively as filled circles and solid vertical lines. Stars show significant difference against 0 and interactions across story types and vigilance states $\left({ }^{\star \star \star} p<0.001,{ }^{\star \star} p<0.01,{ }^{\star} p<0.05\right)$. See also Figures S2A and S2B.

necessarily go against a selective suppression of informative signals during EMs, as meaningless speech presented alone could be treated as informative inputs. It is also unknown whether the selective suppression of informative stories during EMs depends on task instructions, and whether our results would be changed if participants were asked to focus on Jabberwocky stories instead. Further studies are thus necessary to establish how and to what extent internal activity in REM sleep affects the sensory processing of external events.

Finally, our study provides further evidence of a close link between perceptual processing and sleep depth. Using the same approach, we previously found that the selective amplification of informative speech occurs during light NREM sleep but disappears in deep NREM sleep, with a selective suppression of informative speech during slow waves [15]. What we observed in this new study strikingly mirrors these results by showing that the selective amplification of informative speech occurs during tonic REM sleep but disappears during phasic REM sleep, with a selective suppression of informative speech during EMs. Despite the drastic physiological differences between NREM and REM sleep, these results provide a surprisingly coherent picture of the relationship between the selective processing of informative signals and sleep depth.

Overall, our study highlights the role of flexible stimulus selection in the balance between sensory processing and internal activity during REM sleep. The fact that informative stimuli are selectively suppressed, in particular around EMs, buttresses the existence of an informational gating mechanism during REM sleep. Our results provide further support for a distinction between phasic and tonic REM sleep based on the selective gating of informative stimuli and is coherent with reports of poor integration of external stimulation into dreaming activity. Extension of this paradigm to situations where internal activity can be reported (e.g., mind-wandering or using a serial awakening paradigm to probe dreaming activity [33]) would allow us to directly investigate whether the occurrence of internally generated conscious contents is specifically associated with the selective gating of sensory information.

\section{STAR $\star$ METHODS}

Detailed methods are provided in the online version of this paper and include the following:

- KEY RESOURCES TABLE

- RESOURCE AVAILABILITY

○ Lead Contact

O Materials Availability

O Data and Code Availability

- EXPERIMENTAL MODEL AND SUBJECT DETAILS

- METHOD DETAILS

O Stimuli

O Experimental protocol

O EEG recordings

O Sleep scoring

O Stimulus Reconstruction

O Reconstruction around eye movements during REM sleep

- QUANTIFICATION AND STATISTICAL ANALYSIS

\section{SUPPLEMENTAL INFORMATION}

Supplemental Information can be found online at https://doi.org/10.1016/j. cub.2020.04.047.

\section{ACKNOWLEDGMENTS}

This research was supported by ANR grants (ANR-10-LABX-0087 and ANR10-IDEX-0001-02), the European Research Council (ERC project METAWARE to S.K.), the CIFAR (to S.K.), the DGA (to M.K.), and the International Brain Research Organisation (IBRO) and Human Frontier Science Program (HFSP, LT000362/2018-L) to T.A. We thank Shihab Shamma and Daniel Pressnitzer for discussion and Nicolas Obin and Axel Roebel for their help in constructing the stimuli.

\section{AUTHOR CONTRIBUTIONS}

Conceptualization, M.K., C.L., T.A., G.L., and S.K.; Methodology, M.K., C.L., T.A., G.L., and S.K.; Investigation, M.K. and C.L.; Writing - Original Draft, M.K., C.L., T.A., G.L., D.L., and S.D.; Writing - Review \& Editing, M.K., C.L., 
T.A., G.L., D.L., and S.K.; Funding Acquisition, S.K.; Resources, D.L. and S.K.; Supervision, T.A., D.L., and S.K.

\section{DECLARATION OF INTERESTS}

The authors declare no competing interests.

Received: February 8, 2020

Revised: March 20, 2020

Accepted: April 20, 2020

Published: May 14, 2020

\section{REFERENCES}

1. Dement, W., and Wolpert, E.A. (1958). The relation of eye movements, body motility, and external stimuli to dream content. J. Exp. Psychol. 55, 543-553.

2. Rechtschaffen, A., and Foulkes, D. (1965). Effect of visual stimuli on dream content. Percept. Mot. Skills 20, 1149-1160.

3. Berger, R.J. (1963). Experimental modification of dream content by meaningful verbal stimuli. Br. J. Psychiatry 109, 722-740.

4. Nir, Y., and Tononi, G. (2010). Dreaming and the brain: from phenomenology to neurophysiology. Trends Cogn. Sci. 14, 88-100.

5. Issa, E.B., and Wang, X. (2008). Sensory responses during sleep in primate primary and secondary auditory cortex. J. Neurosci. 28, 14467-14480.

6. Nir, Y., Vyazovskiy, V.V., Cirelli, C., Banks, M.I., and Tononi, G. (2015). Auditory responses and stimulus-specific adaptation in rat auditory cortex are preserved across NREM and REM sleep. Cereb. Cortex 25, 13621378.

7. Perrin, F., García-Larrea, L., Mauguière, F., and Bastuji, H. (1999). A differential brain response to the subject's own name persists during sleep. Clin. Neurophysiol. 110, 2153-2164.

8. Blume, C., Del Giudice, R., Wislowska, M., Heib, D.P.J., and Schabus, M. (2018). Standing sentinel during human sleep: continued evaluation of environmental stimuli in the absence of consciousness. Neuroimage $178,638-648$.

9. Strauss, M., Sitt, J.D., King, J.R., Elbaz, M., Azizi, L., Buiatti, M., Naccache, L., van Wassenhove, V., and Dehaene, S. (2015). Disruption of hierarchical predictive coding during sleep. Proc. Natl. Acad. Sci. USA 112, E1353-E1362.

10. Dement, W., and Kleitman, N. (1957). The relation of eye movements during sleep to dream activity: an objective method for the study of dreaming. J. Exp. Psychol. 53, 339-346.

11. Goodenough, D.R., Shapiro, A., Holden, M., and Steinschriber, L. (1959). A comparison of" dreamers" and" nondreamers": Eye movements, electroencephalograms, and the recall of dreams. J. Abnorm. Soc. Psychol. 59, 295.

12. Ermis, U., Krakow, K., and Voss, U. (2010). Arousal thresholds during human tonic and phasic REM sleep. J. Sleep Res. 19, 400-406.

13. Mesgarani, N., and Chang, E.F. (2012). Selective cortical representation of attended speaker in multi-talker speech perception. Nature 485, 233-236.

14. O'Sullivan, J.A., Power, A.J., Mesgarani, N., Rajaram, S., Foxe, J.J., Shinn-Cunningham, B.G., Slaney, M., Shamma, S.A., and Lalor, E.C. (2015). Attentional selection in a cocktail party environment can be decoded from single-trial EEG. Cereb. Cortex 25, 1697-1706.

15. Legendre, G., Andrillon, T., Koroma, M., and Kouider, S. (2019). Sleepers track informative speech in a multitalker environment. Nat. Hum. Behav. 3, 274-283.

16. Spreng, L.F., Johnson, L.C., and Lubin, A. (1968). Autonomic correlates of eye movement bursts during stage REM sleep. Psychophysiology 4 , 311-323.

17. Arnulf, I. (2011). The 'scanning hypothesis' of rapid eye movements during REM sleep: a review of the evidence. Arch. Ital. Biol. 149, 367-382.
18. Bastuji, H., Perrin, F., and Garcia-Larrea, L. (2002). Semantic analysis of auditory input during sleep: studies with event related potentials. Int. J. Psychophysiol. 46, 243-255.

19. Tamaki, M., and Sasaki, Y. (2019). Surveillance during REM sleep for the first-night effect. Front. Neurosci. 13, 1161.

20. Sallinen, M., Kaartinen, J., and Lyytinen, H. (1996). Processing of auditory stimuli during tonic and phasic periods of REM sleep as revealed by eventrelated brain potentials. J. Sleep Res. 5, 220-228.

21. Wehrle, R., Kaufmann, C., Wetter, T.C., Holsboer, F., Auer, D.P. Pollmächer, T., and Czisch, M. (2007). Functional microstates within human REM sleep: first evidence from fMRI of a thalamocortical network specific for phasic REM periods. Eur. J. Neurosci. 25, 863-871.

22. McCormick, D.A., and Bal, T. (1994). Sensory gating mechanisms of the thalamus. Curr. Opin. Neurobiol. 4, 550-556.

23. Esser, S.K., Hill, S., and Tononi, G. (2009). Breakdown of effective connectivity during slow wave sleep: investigating the mechanism underlying a cortical gate using large-scale modeling. J. Neurophysiol. 102, 2096-2111.

24. Andrillon, T., and Kouider, S. (2019). The vigilant sleeper: neural mechanisms of sensory (de) coupling during sleep. Curr. Opin. Physiol. Published online 13 December 2019. https://doi.org/10.1016/j.cophys.2019.12.002.

25. Ding, N., and Simon, J.Z. (2012). Emergence of neural encoding of auditory objects while listening to competing speakers. Proc. Natl. Acad. Sci. USA 109, 11854-11859.

26. Hong, C.C.H., Fallon, J.H., Friston, K.J., and Harris, J.C. (2018). Rapid eye movements in sleep furnish a unique probe into consciousness. Front. Psychol. 9, 2087.

27. Leclair-Visonneau, L., Oudiette, D., Gaymard, B., Leu-Semenescu, S., and Arnulf, I. (2010). Do the eyes scan dream images during rapid eye movement sleep? Evidence from the rapid eye movement sleep behaviour disorder model. Brain 133, 1737-1746.

28. Doricchi, F., laria, G., Silvetti, M., Figliozzi, F., and Siegler, I. (2007). The "ways" we look at dreams: evidence from unilateral spatial neglect (with an evolutionary account of dream bizarreness). Exp. Brain Res. 178, 450-461.

29. Hong, C.C.H., Harris, J.C., Pearlson, G.D., Kim, J.S., Calhoun, V.D. Fallon, J.H., Golay, X., Gillen, J.S., Simmonds, D.J., van Zijl, P.C.M. et al. (2009). fMRI evidence for multisensory recruitment associated with rapid eye movements during sleep. Hum. Brain Mapp. 30, 1705-1722.

30. Miyauchi, S., Misaki, M., Kan, S., Fukunaga, T., and Koike, T. (2009). Human brain activity time-locked to rapid eye movements during REM sleep. Exp. Brain Res. 192, 657-667.

31. Andrillon, T., Nir, Y., Cirelli, C., Tononi, G., and Fried, I. (2015). Singleneuron activity and eye movements during human REM sleep and awake vision. Nat. Commun. 6, 7884.

32. Ogawa, K., Nittono, H., and Hori, T. (2005). Brain potentials before and after rapid eye movements: an electrophysiological approach to dreaming in REM sleep. Sleep 28, 1077-1082.

33. Siclari, F., Larocque, J.J., Postle, B.R., and Tononi, G. (2013). Assessing sleep consciousness within subjects using a serial awakening paradigm. Front. Psychol. 4, 542.

34. Stickgold, R., Malia, A., Fosse, R., Propper, R., and Hobson, J.A. (2001) Brain-mind states: I. Longitudinal field study of sleep/wake factors influencing mentation report length. Sleep 24, 171-179.

35. Siclari, F., Baird, B., Perogamvros, L., Bernardi, G., LaRocque, J.J., Riedner, B., Boly, M., Postle, B.R., and Tononi, G. (2017). The neural correlates of dreaming. Nat. Neurosci. 20, 872-878.

36. Chow, H.M., Horovitz, S.G., Carr, W.S., Picchioni, D., Coddington, N., Fukunaga, M., Xu, Y., Balkin, T.J., Duyn, J.H., and Braun, A.R. (2013). Rhythmic alternating patterns of brain activity distinguish rapid eye movement sleep from other states of consciousness. Proc. Natl. Acad. Sci. USA 110, 10300-10305.

37. Llinás, R.R., and Paré, D. (1991). Of dreaming and wakefulness. Neuroscience 44, 521-535. 
38. Formby, D. (1967). Maternal recognition of infant's cry. Dev. Med. Child Neurol. 9, 293-298.

39. Obin, N. (2011). MeLos: analysis and modelling of speech prosody and speaking style. PhD thesis (Université Pierre et Marie Curie - Paris VI).

40. Leclercq, Y., Schrouff, J., Noirhomme, Q., Maquet, P., and Phillips, C. (2011). fMRI artefact rejection and sleep scoring toolbox. Comput. Intell. Neurosci. 2011, 598206.

41. Oostenveld, R., Fries, P., Maris, E., and Schoffelen, J.M. (2011). FieldTrip: Open source software for advanced analysis of MEG, EEG, and invasive electrophysiological data. Comput. Intell. Neurosci. 2011, 156869.
42. Delorme, A., and Makeig, S. (2004). EEGLAB: an open source toolbox for analysis of single-trial EEG dynamics including independent component analysis. J. Neurosci. Methods 134, 9-21.

43. Krekelberg, K. (2019). bayesFactor, GitHub. https://www.github.com/ klabhub/bayesFactor.

44. Iber, C., and Iber, C. (2007). The AASM Manual for the Scoring of Sleep and Associated Events: Rules, Terminology and Technical SpecificationsVolume 1 (American Academy of Sleep Medicine). 


\section{STAR $\star M E T H O D S$}

\section{KEY RESOURCES TABLE}

\begin{tabular}{|c|c|c|}
\hline REAGENT or RESOURCE & SOURCE & IDENTIFIER \\
\hline \multicolumn{3}{|l|}{ Software and Algorithms } \\
\hline MATLAB, Matlab2019a & Mathworks, Natick, MA, USA & https://www.mathworks.com \\
\hline Logic Pro software, IRCAMTRAX module & Apple, Cupertino, CA, USA & https://www.apple.com/fr/logic-pro/ \\
\hline $\begin{array}{l}\text { IRCAMTTS, MATLAB-based text-to-speech } \\
\text { algorithm }\end{array}$ & {$[39]$} & $\begin{array}{l}\text { http://anasynth.ircam.fr/home/english/software/ } \\
\text { ircamtts-en }\end{array}$ \\
\hline FASST, MATLAB-based sleep scoring toolbox & {$[40]$} & $\begin{array}{l}\text { http://www.montefiore.ulg.ac.be/ phillips/FASST. } \\
\text { html }\end{array}$ \\
\hline Fieldtrip, MATLAB-based EEG analysis toolbox & {$[41]$} & http://www.fieldtriptoolbox.org \\
\hline EEGLAB, MATLAB-based EEG analysis toolbox & {$[42]$} & https://sccn.ucsd.edu/eeglab/index.php \\
\hline bayesFactor, MATLAB based statistical toolbox & [43] & https://www.github.com/klabhub/bayesFactor \\
\hline \multicolumn{3}{|l|}{ Other } \\
\hline Echo Fire 12 Soundcard & $\begin{array}{l}\text { Echo Digital Audio, Santa Barbara, } \\
\text { CA, USA }\end{array}$ & https://echoaudio.com/ \\
\hline $\begin{array}{l}64 \text { channels EGI EEG recording system, NetAmp } \\
300\end{array}$ & Electrical Geodesic & https://www.egi.com \\
\hline $\begin{array}{l}\text { non-electrical earplugs RLINK Ear Tone 3A, } 10 \\
\text { Ohms }\end{array}$ & Interacoustic & https://www.interacoustics.com/ \\
\hline
\end{tabular}

\section{RESOURCE AVAILABILITY}

\section{Lead Contact}

Further information and requests should be directed to and will be fulfilled by the Lead Contact, Sid Kouider (sid.kouider@ens.fr).

Materials Availability

The stimuli that support the findings of this study are available from the corresponding author upon reasonable request.

Data and Code Availability

The data that support the findings of this study are available from the corresponding author upon reasonable request.

\section{EXPERIMENTAL MODEL AND SUBJECT DETAILS}

42 French-native speakers (mean: 25.1 years old, min: 18, max: 31, 17 females) with self-declared normal hearing and no history of sleep disorders were recruited for this study. Participant sample size was chosen based on previous results using the same methodology [15]. Easy sleepers (Epworth Sleepiness Score, mean: 12.5, min: 10, max: 15) with normal or evening chronotypes (Morningness-eveningness score, mean: 42.1, min: 31, max: 54) were selected through online questionnaires to facilitate propensity to fall asleep in the morning. To increase sleepiness and ensure high REM sleep pressure during the experiment, participants were required to sleep about $30 \%$ less during the night preceding the experiment (verified with actimetry) and to arrive at 7.30 a.m. at the sleep laboratory. They were also deprived from stimulants (e.g., caffeine) the day of the experiment. The present protocol has been approved by the local ethical committee (Comité de Protection des Personnes, lle-de-France I, Paris, France) and all participants provided informed consent.

\section{METHOD DETAILS}

Stimuli

Stimuli used for this study were identical to Legendre and colleagues [15]. Eighty meaningful (informative) and meaningless (Jabberwocky) stories, i.e., texts for which content words (e.g., nouns and verbs) were replaced by French pseudo-words, and matched in length, syntax, word-frequency and phonemic properties. Stories were generated using IRCAMTTS, a state-of-the-art Text-tospeech MATLAB software [39]. Using the IRCAMTRAX module of Logic Pro software (Apple), we then manipulated the voice to generate two copies of each audio story, one pronounced by a low-pitched voice and one by a high-pitched voice. Eighty pairs of informative and Jabberwocky were then formed and matched in duration and acoustic properties $(73.57 \pm 5.16 \mathrm{~s}$, mean $\pm \mathrm{SD}$ 
across stories, min: 54.04 s, max: 83.51 s). For the Training phase, the same text was played in both ears. For the Test phase, a pair of Jabberwocky and informative stories were played each in a different ear and in a different pitch. The side of the stimulation (left or right ear) and the pitch of the voice (low and high pitch) of the informative speech was randomized trial-by-trial and counterbalanced for each pair across participants. Stimuli were delivered to participants using non-electrical earplugs (RLINK Ear Tone 3A, 10 Ohms, Interacoustic) and played using an Echo Fire 12 Soundcard (Echo Digital Audio, Santa Barbara, CA, USA). The volume of the stimulation was set around $50 \mathrm{~dB}$ and adapted to participant's preferences, in line with previous studies [9, 15].

\section{Experimental protocol}

During the entire experiment, participants laid down in a dark, isolated room and were equipped with 64-channel EEG gel-nets (EGI system, Electrical Geodesic) and chin-EMG. First, subjects had a Training phase (duration: 12 minutes) during which they were presented with 6 informative stories followed by 6 Jabberwocky stories played diotically (i.e., with the same story played in both ears). Next, they went through the first part of the Test phase during which they were exposed to 8 trials played dichotically (i.e., one different stream in each ear, informative and Jabberwocky). Participants were instructed to focus exclusively on the informative speech while ignoring the Jabberwocky one. During both Training and the first part of the Test phase, participants were instructed to stay awake with their eyes closed to avoid eye movement artifacts and keep the same conditions throughout the experiment. Then, subjects entered the second part of the testing phase during which participants were continuously exposed to the same 8 trials. Participants were instructed to pursue the task with the additional instruction that they could fall asleep from now on. Sleep onset was assessed online and defined following standard guidelines as the first occurrence of spindles or spontaneous K-complexes [44]. Following the visual detection of sleep onset by trained scorers (MK and $\mathrm{CL}$ ), trials were replaced by new pairs of informative and Jabberwocky stories played dichotically to the sleeping subjects. During the Training phase, trials were self-paced while during the Test phase, trials were separated with jitters of 4 to $6 \mathrm{~s}$ (random uniform distribution). If participants did not manage to fall asleep after 45 minutes, the experiment was terminated.

\section{EEG recordings}

EEG signals were amplified (NetAmp 300), referenced online to $\mathrm{Cz}$ and sampled at $500 \mathrm{~Hz}$. Electrooculograms (EOG) and chin electromyograms (EMG) were recorded with electrodes placed respectively around the eyes and on the chin. EEG recordings and audio stimuli were synchronized using a third audio channel through which a tone was played at the onset and end of each trial and recorded by the amplifier (NetAmp 300).

\section{Sleep scoring}

\section{sleep scoring}

Vigilance states were scored offline on 20 s-long windows by trained scorers (MK and CL) and confirmed by an expert scorer (DL) following established guidelines [44] and using the FASST toolbox [40]. EEG and EOG signals were re-referenced to mastoids and bandpass filtered between 0.1 and $30 \mathrm{~Hz}$ (two-pass Butterworth filter, 5th order). EMG signal was obtained with a local derivation and bandpassed between 80 and $160 \mathrm{~Hz}$ (two-pass Butterworth filter, 5th order). For the sleeping Test phase, only novel trials containing novel stimuli (i.e., not played during wakefulness) were kept for analysis. NREM1 was excluded from analyses as it is considered as a transitory state between wake and sleep. Trials that contained a mixture of vigilance states were excluded from analysis. Participants with less than 4 trials within REM sleep were discarded from further analyses, resulting in conserving 18 out of 42 participants (number of trials in REM sleep, mean $=10.8 \pm 1.5$ ). Table S1 summarizes the sleep statistics across subjects.

Detection of rapid eye movements

Eye movements (EMs) were visually identified by trained scorers (MK and CL) blind to experimental conditions. To do so, EOG derivations were extracted from EEG signals around eye movements and were re-referenced to the contralateral mastoid. EOG signals were bandpass filtered between 0.5 and $2 \mathrm{~Hz}$ (two-pass Butterworth filter, 5th order). Co-ocurrent deviations in left and right EOG were visually detected and the onset of the first deviation was marked as EM onset. EMs were further visually classified into two categories: "isolated" when EMs were followed by no more than one EM and "burst" when EMs were followed or preceded by at least two other EMs. To analyze more carefully the effect of burst of EMs on speech processing, we further distinguished the onset of bursts, defined as the absence of bursts in the 12 preceding seconds, and the offset of bursts, defined as the absence of burst in the 12 successive seconds. Finally, we redefined our scoring windows of $20 \mathrm{~s}$ as phasic REM sleep if they contained burst EMs, and tonic REM sleep otherwise. Subjects with more than 4 windows were conserved for analysis, resulting in 18 subjects for tonic REM sleep and 8 for phasic REM sleep.

\section{Stimulus Reconstruction}

Preprocessing

The EEG signal was first re-referenced to the average of all sensors and then high-pass filtered at $0.5 \mathrm{~Hz}$ and low-pass filtered at $8 \mathrm{~Hz}$ with a two-pass Butterworth filter ( $5^{\text {th }}$ order) using the fieldtrip toolbox [41]. The filtered EEG signal was aligned with auditory signals. The sound envelope of each auditory stream of the corresponding trial was obtained by applying the Hilbert transform and filtered below $8 \mathrm{~Hz}$ with a two-pass Butterworth filter ( $5^{\text {th }}$ order). Both EEG and auditory signals were then down-sampled at $100 \mathrm{~Hz}$. 
Training the linear model on diotic trials

The stimulus reconstruction approach relies on two phases. The Training phase consists in building an optimal linear model of the brain response (here the EEG envelope of the 64 channels) to stimulation (here the auditory envelope). This model was trained on diotic trials of the Training phase (6 informative stories then 6 Jabberwocky stories played in both ears) to ensure that it was independent from selective processing and story type. Because the brain processes auditory signals with different time delays (referred to as time-lags), we first identified the range of time-lags during which auditory signals are processed. To do so, we first extracted the detrended EEG data. Using trials from the Training phase, we reconstructed the auditory input at individual time-lags using a leaveone-out procedure (i.e., training the model on 11 trials and testing on the remaining trial, successively for every trial of the Training phase of each participant). The reconstruction score for each trial and each time-lag was defined as the Pearson's correlation between the envelope reconstructed from the EEG signal and the envelope of the acoustic input. The reconstruction scores were averaged within participants for each time-lag and compared to 0 . The cluster of time-lags with reconstruction scores significantly above 0 ranged from $-190 \mathrm{~ms}$ to $840 \mathrm{~ms}$ and was selected for subsequent analyses (Figure S1A). We also verified that the model was not biased toward any stimulus category. To do so, the stimulus reconstruction was performed separately for informative and Jabberwocky speech using the aforementioned leave-one-out procedure. Reconstruction scores were averaged per stimulus category and across participants (Figure S1B).

Testing on dichotic trials

The second phase consists in using the model trained on the Training phase to reconstruct an auditory envelope from the brain activity during the Test trials. This envelope was compared to the envelope of each auditory stream using a Pearson's correlation. A correlation score here called reconstruction score was obtained for each type of speech: $r_{\text {informative }}$ and $r_{\text {Jabberwocky }}$. For each trial, attention was defined as correct, i.e., being oriented toward the informative speech, if $r_{\text {informative }}$ was higher than $r_{\text {Jabberwocky }}$ (i.e., the reconstructed envelope was closer to the envelope of the informative speech). The percentage of trials with a higher reconstruction score for the informative speech compared to the Jabberwocky speech refers to the decoding performance (\%). To compare tonic and phasic REM sleep, reconstruction scores were obtained on scoring windows of $20 \mathrm{~s}$.

Reconstruction around eye movements during REM sleep

Artifact removal with ICA

Independent components corresponding to eye movement artifacts during REM sleep were first identified using ICA in EEGLab [42]. Visually-selected components were removed from the EEG signal.

Reconstruction

The influence of physiological events (i.e., EMs) on the processing of each auditory stream was investigated by computing the reconstruction score of each stream on sliding windows of $4 \mathrm{~s}$ centered on the onset of EMs $(-10 \mathrm{~s}$ to $+10 \mathrm{~s}$ with $100 \mathrm{~ms}$ steps, see Figures $3 \mathrm{C}$ and S3). Time-courses of the reconstruction scores were averaged across participants, including participants with at least 4 detected micro-events ( $\mathrm{N}=14$ for all and isolated EMs, 7 for the onset and offset of bursts). Time-courses were smoothed for visualization purposes only using a 500-ms-wide Gaussian kernel, but statistical tests were performed on the reconstruction scores before smoothing. Results were averaged across participants.

\section{QUANTIFICATION AND STATISTICAL ANALYSIS}

Pearson's R was used as a parametric measure of the correlation between the reconstructed envelope and the auditory stimuli, resulting in a reconstruction score for each stream [15]. Reconstruction scores between speech streams and across sleep stages, as well as decoding performance across sleep stages, were compared using non-parametric paired statistics (Wilcoxon signed rank test). Bonferroni correction for multiple comparisons was applied for post hoc tests. Effect sizes calculated following the formula: $r=Z / \sqrt{ } n$, where $Z$ is the $z$-stats of the Wilcoxon signed rank test and $n$ the number of data-points. In case of non-significant results, bayesian statistics were computed using the bayesFactor toolbox in MATLAB [43]. A Bayes Factor above 3 typically provides supportive evidence for the null hypothesis. Linear mixed-effect models were performed using MATLAB fitlme function to evaluate the interactions between experimental conditions on reconstruction scores and decoding performances. This technique allows comparisons between unbalanced datasets among conditions and across participants with heterogeneous sleep patterns. All dependent variables were defined as categorical values. When comparing time-series of reconstruction scores, we relied on non-parametric cluster permutation statistics to control for multiple comparisons. Clusters were defined as consecutive time-points for which parametric $t$ tests reached a specific threshold $(\alpha=0.05)$. For each cluster, the sum of $t$-values was compared to the maximum cluster statistics obtained after random permutation of the conditions considered ( $n=1000$ permutations). We computed a Monte-Carlo $P$ value (referred to as $P_{\text {cluster}}$ ). For each significant cluster, the average Cohen's d (mean over standard deviation) was calculated for each participant and was reported as the effect-size. 